\title{
A SPREAD RELATION FOR ENTIRE FUNCTIONS WITH NEGATIVE ZEROS
}

\author{
FARUK F. ABI-KHUZAM
}

(Communicated by Clifford J. Earle, Jr.)

\begin{abstract}
Let $g$ be a canonical product having only real negative zeros and nonintegral order $\lambda$, and let $\phi$ be the set function defined by $2 \pi \phi(E)=$ $\int_{E} \pi \lambda \csc \pi \lambda \cos \lambda \theta d \theta$. It is shown that if $E(r)$ is the set of values of $\theta \in$ $(-\pi, \pi]$ where $\left|g\left(r e^{i \theta}\right)\right| \geq 1, r_{n}$ is a sequence of Polya peaks of $g$ and $\delta$ is the deficiency of the value zero of $g$ then $\liminf \phi\left(E\left(r_{n}\right)\right) \geq 2(1-\delta)^{-1}$. This inequality leads to a sharp spread relation for $g$.
\end{abstract}

\section{INTRODUCTION}

Let

$$
g(z)=\prod_{n=1}^{\infty}\left(1-z / a_{n}\right) \exp \left(\sum_{j=1}^{q} \frac{1}{j}\left(z / a_{n}\right)^{j}\right)
$$

denote a Weierstrass canonical product of genus $q \geq 1$, having only negative zeros $a_{n}$. Denote by

$$
T(r, g)=\frac{1}{2 \pi} \int_{-\pi}^{\pi} \log ^{+}\left|g\left(r e^{i \theta}\right)\right| d \theta
$$

the Nevanlinna characteristic of $g$, and denote by

$$
\mu=\liminf _{r \rightarrow \infty} \frac{\log T(r, g)}{\log r}
$$

the lower order of $g$.

Let

$$
E(r)=\left\{\theta \in[-\pi, \pi]: \log \left|g\left(r e^{i \theta}\right)\right| \geq 0\right\},
$$

and denote by $|E(r)|$ the measure of the Lebesgue measurable set $E(r)$. Then it is a consequence of the spread relation for meromorphic functions conjectured by Edrei and proved by Baernstein [3] that

$$
\liminf _{m \rightarrow \infty}\left|E\left(r_{m}\right)\right| \geq \frac{\pi}{\mu},
$$

Received by the editors June 15, 1989.

1980 Mathematics Subject Classification (1985 Revision). Primary 30D20, 30D15; Secondary 30D35. 
where $r_{m}$ is a sequence of Polya peaks of order $\mu$ of $T(r, g)$. The exact meaning of these peaks will not be needed here; for their definition see [4]. In private communication, Professor Daniel Shea asked whether (5) can be improved for the functions $g$ defined by (1) and having only negative zeros. It is the purpose of this note to obtain a sharp form of (5) for such functions.

Recall that the deficiency of the value 0 of $g$ is defined by

$$
\delta(0)=\delta(0, g)=1-\limsup _{r \rightarrow \infty} \frac{N(r, 1 / g)}{T(r, g)},
$$

where

$$
N(r, 1 / g)=\int_{0}^{r} t^{-1} n(t) d t
$$

and $n(t)$ is the number of zeros of $g$ in the disk $|z| \leq t$. Our results may be stated as follows:

Theorem 1. Let $g$ be a Weierstrass canonical product of genus $q \geq 1$, having only negative zeros and assume that the lower order $\mu$ of $g$ is nonintegral. Let $\psi_{\mu}$ be the function defined on $(-\pi, \pi)$ by

$$
\psi_{\mu}(\theta)=\pi \mu \csc \pi \mu \cos \mu \theta,
$$

and introduce the set function $\phi$ defined on Lebesgue measurable subsets of $[-\pi, \pi]$ by

$$
\phi(E)=\frac{1}{\pi} \int_{E} \psi_{\mu}(\theta) d \theta .
$$

If $r_{m}$ is a sequence of Polya peaks of order $\mu$ of $T(r, g)$ and $\delta(0)$ is the deficiency of the value 0 of $g$, then

$$
\liminf _{m \rightarrow \infty} \phi\left(E\left(r_{m}\right)\right) \geq \frac{2}{1-\delta(0)}
$$

and

$$
\liminf _{m \rightarrow \infty}\left|E\left(r_{m}\right)\right| \geq \frac{2(q+1)}{\mu} \sin ^{-1}\left\{\frac{|\sin \pi \mu|}{(q+1)(1-\delta(0))}\right\} .
$$

Furthermore, both inequalities are sharp.

If the lower order of $g$ satisfies $q<\mu \leq q+\frac{1}{2}$, then it may happen that one component of the set $E\left(r_{m}\right)$ extends all the way up to $\pi$. In this case we can obtain a sharper form of (11):

Theorem 2. Let $g$ be as in Theorem 1 and assume that

$$
q<\mu \leq q+\frac{1}{2} \text {. }
$$

If $\pi \in E\left(r_{m}\right)$ for infinitely many values of $m$, then

$$
\limsup _{m \rightarrow \infty}\left|E\left(r_{m}\right)\right| \geq 2 \pi(1-q / \mu)+\frac{2 q}{\mu} \sin ^{-1}\left\{\frac{|\sin \pi \mu| \delta(0)}{q(1-\delta(0))}\right\} .
$$

Furthermore, (13) is sharp. 
For the case $0<\mu<1$, the analogues of (10) and (11) continue to hold true [4] and require no geometric restriction on the zeros of $g$. As for (13), the spread relation of Edrei gives the stronger result $\liminf \left|E\left(r_{m}\right)\right|=2 \pi$.

An entire function having finite lower order $\mu$ and having all its zeros on one ray necessarily [2] has finite order $\lambda$ and satisfies $\lambda \leq[\mu]+1$. Thus for the functions $g$ defined by (1) we always have $q \leq \mu \leq \lambda \leq q+1$. The assumptions in Theorems 1 and 2 imply $q<\mu<q+1$.

Our proofs depend on the following two results of Hellerstein and Williamson [5]:

Theorem A. Let $g$ be as in the statement of Theorem 1 and let

$$
C(r)=\left\{\theta \in[0, \pi]: \log \left|g\left(r e^{i \theta}\right)\right| \geq 0\right\} .
$$

Then for each $r>0$, there exist points $\alpha_{1}=\alpha_{1}(r), \ldots, \alpha_{q+1}=\alpha_{q+1}(r)$ satisfying

$$
\frac{2 j-1}{2(q+1)} \pi<\alpha_{j}<\frac{2 j-1}{2 q} \pi \quad(j=1,2, \ldots, q)
$$

and

$$
\frac{2 q+1}{2(q+1)} \pi<\alpha_{q+1} \leq \pi,
$$

such that

$$
C(r)=\bigcup_{j=1}^{(q+1) / 2}\left[\alpha_{2 j-1}, \alpha_{2 j}\right] \text { if } q \text { is odd }
$$

and

$$
C(r)=\bigcup_{j=0}^{q / 2}\left[\alpha_{2 j}, \alpha_{2 j+1}\right], \alpha_{0}=0 \quad \text { if } q \text { is even. }
$$

Theorem B. Let $g$ be as in Theorem 1 and let $r_{m}$ be a sequence of Polya peaks of order $\mu$ of $T(r, g)$. If

$$
\sigma>\limsup _{r \rightarrow \infty} \frac{N(r, 1 / g)}{T(r, g)},
$$

then

$$
1 \leq \sigma(1+o(1))|\csc \pi \mu| \sum_{j=0}^{[(q+1) / 2]}\left(\sin \mu \alpha_{2 j+1}-\sin \mu \alpha_{2 j}\right)+o(1)
$$

as $m \rightarrow \infty$, where $\alpha_{q+2}=0$.

The inequality in (20) above is a restatement of the inequality (4.17) in [5, p. 249] where we have divided by $T\left(r_{m}, g\right)$. The dependence on the Polya peaks occurs in $\alpha_{j}=\alpha_{j}\left(r_{m}\right)$. 


\section{Proof of Theorem 1}

The sets $E(r)$ and $C(r)$ defined in (4) and (14) respectively satisfy $E(r)=$ $C(r) \cup-C(r)$. This is because the function $\left|g\left(r e^{i \theta}\right)\right|$ is an even function of $\theta$, a fact which follows from (1) and the reality of the zeros $a_{n}$. Recalling the definitions of $\psi_{\mu}(\theta)$ and $\phi$ we have

$$
\frac{1}{2} \phi\left(E\left(r_{m}\right)\right)=\frac{1}{\pi} \int_{C\left(r_{m}\right)} \psi_{\mu}(\theta) d \theta .
$$

If $q$ is odd, then by (17) the integral in (2.1) equals

$$
\csc \pi \mu \sum_{j=1}^{(q+1) / 2}\left(\sin \mu \alpha_{2 j}-\sin \mu \alpha_{2 j-1}\right)=|\csc \pi \mu| \sum_{j=0}^{[(q+1) / 2]}\left(\sin \mu \alpha_{2 j+1}-\sin \mu \alpha_{2 j}\right),
$$

where we have used $\alpha_{0}=\alpha_{q+2}=0$ and $q<\mu<q+1$ to write $\csc \pi \mu=$ $-|\csc \pi \mu|$. If $q$ is even, then in view of (18) the integral in (2.1) equals

$$
\csc \pi \mu \sum_{j=0}^{q / 2}\left(\sin \mu \alpha_{2 j+1}-\sin \mu \alpha_{2 j}\right)=|\csc \pi \mu| \sum_{j=0}^{[(q+1) / 2]}\left(\sin \mu \alpha_{2 j+1}-\sin \mu \alpha_{2 j}\right) .
$$

Using (20) and the above, we obtain

$$
2 \leq \sigma(1+o(1)) \phi\left(E\left(r_{m}\right)\right)+o(1) \text { as } m \rightarrow \infty .
$$

Now (10) follows immediately from (2.2), (19), and (6).

We note here that (2.2) and (2.1) imply

$$
\begin{aligned}
2 & \leq \sigma(1+o(1)) \phi\left(E\left(r_{m}\right)\right)+o(1) \\
& \leq \sigma(1+o(1)) \times \frac{2}{\pi} \int_{0}^{\pi} \psi_{\mu}^{+}(\theta) d \theta+o(1) \quad \text { as } m \rightarrow \infty .
\end{aligned}
$$

The first and last inequalities imply that

$$
\liminf _{r \rightarrow \infty} \frac{T(r, g)}{N(r, 1 / g)} \leq \frac{1}{\pi} \int_{0}^{\pi} \psi_{\mu}^{+}(\theta) d \theta,
$$

which is the well-known result of Hellerstein and Williamson [5]. Note that (2.3) may be used to obtain information about functions extremal for the inequality (2.4). Indeed, if equality holds in (2.4), then (2.3) implies that $\phi\left(E\left(r_{m}\right)\right) \rightarrow$ $\frac{1}{\pi} \int_{0}^{\pi} \psi_{\mu}^{+}(\theta) d \theta$ as $m \rightarrow \infty$, and we may conclude from this that

$$
\alpha_{j}\left(r_{m}\right) \rightarrow \frac{(2 j-1)}{2 \mu} \pi \quad \text { as } m \rightarrow \infty,
$$

for $j=1, \ldots, q+1$ if $q+\frac{1}{2}<\mu \leq q+1$, and for $j=1, \ldots, q$ and $\alpha_{q+1} \rightarrow \pi$ if $q<\mu \leq q+\frac{1}{2}$. 
Proof of (11). Suppose first that $q$ is odd. Using the elementary identity $\sin x-$ $\sin y=2 \cos \left(\frac{x+y}{2}\right) \sin \left(\frac{x-y}{2}\right)$, we obtain

$$
\begin{aligned}
\mid \csc & \pi \mu \mid \sum_{j=0}^{[(q+1) / 2]}\left(\sin \mu \alpha_{2 j+1}-\sin \mu \alpha_{2 j}\right) \\
& =|\csc \pi \mu| \sum_{j=1}^{q+1}(-1)^{j-1} \sin \mu \alpha_{j} \\
& =2|\csc \pi \mu| \sum_{j=1}^{(q+1) / 2} \sin \frac{1}{2} \mu\left(\alpha_{2 j-1}-\alpha_{2 j}\right) \cos \frac{1}{2} \mu\left(\alpha_{2 j-1}+\alpha_{2 j}\right) \\
& \leq 2|\csc \pi \mu| \sum_{j=1}^{(q+1) / 2} \sin \frac{1}{2} \mu\left(\alpha_{2 j}-\alpha_{2 j-1}\right) .
\end{aligned}
$$

To justify the last inequality in (2.5), we must show that $\frac{1}{2} \mu\left(\alpha_{2 j}-\alpha_{2 j-1}\right) \leq \pi$ for $j=1,2, \ldots,(q+1) / 2$. By (15) and (16) we have $\alpha_{q+1}-\alpha_{q} \leq \pi-\frac{2 q-1}{2(q+1)} \pi=$ $\frac{3 \pi}{2(q+1)}<\frac{2 \pi}{\mu}$ since $q<\mu<q+1$. Also, if $q>1$, then for $j=1, \ldots,(q-1) / 2$ we have by $(15)$

$$
\begin{aligned}
\alpha_{2 j}-\alpha_{2 j-1} & \leq \frac{4 j-1}{2 q} \pi-\frac{4 j-3}{2(q+1)} \pi=\frac{4 j+2 q-1}{2 q(q+1)} \pi \\
& \leq \frac{4 q-3}{2 q(q+1)} \pi<2 \pi /(q+1)<2 \pi / \mu .
\end{aligned}
$$

Thus in all cases $\frac{1}{2} \mu\left(\alpha_{2 j}-\alpha_{2 j-1}\right) \leq \pi$ and the inequality in (2.5) is justified.

To continue, note that the function $\sin x$ is concave on $[0, \pi]$ since $(\sin x)^{\prime \prime}$ $=-\sin x \leq 0$ for all $x \in[0, \pi]$. Thus $\sin \left(n^{-1} \sum_{j=1}^{n} x_{j}\right) \geq n^{-1} \sum_{j=1}^{n} \sin x_{j}$ for any $n$ numbers $x_{j} \in[0, \pi]$. Taking $x_{j}=\frac{1}{2} \mu\left(\alpha_{2 j}-\alpha_{2 j-1}\right)$ where $j=$ $1, \ldots,(q+1) / 2$, we obtain

$$
\begin{aligned}
\sum_{j=1}^{(q+1) / 2} \sin \frac{1}{2} \mu\left(\alpha_{2 j}-\alpha_{2 j-1}\right) & \leq \frac{q+1}{2} \sin \left\{\frac{2}{q+1} \sum_{j=1}^{(q+1) / 2} \frac{1}{2} \mu\left(\alpha_{2 j}-\alpha_{2 j-1}\right)\right\} \\
& =\frac{(q+1)}{2} \sin \left\{\frac{\mu}{q+1}\left|C\left(r_{m}\right)\right|\right\},
\end{aligned}
$$

where $\left|C\left(r_{m}\right)\right|$ is the measure of the set $C\left(r_{m}\right)$ defined in (14), (17). Now (20), (2.5), and (2.6) imply

$$
1 \leq \sigma(1+o(1))\left\{\frac{(q+1)}{|\sin \pi \mu|} \sin \left(\frac{\mu}{q+1}\left|C\left(r_{m}\right)\right|\right)\right\}+o(1)
$$

as $m \rightarrow \infty$.

We may assume that for all large $m,\left|C\left(r_{m}\right)\right|<(q+1) \pi / 2 \mu$, since otherwise the inequality in (11) is manifestly true. Thus $(2.7)$ now implies that

$$
\left|C\left(r_{m}\right)\right| \geq \frac{q+1}{\mu} \sin ^{-1}\left\{\frac{|\sin \pi \mu|}{(q+1)} \cdot \frac{(1+o(1))}{\sigma}\right\} \quad(m \rightarrow \infty) .
$$


Recalling (19) and (6), we see that (2.8) leads to

$$
\liminf _{m \rightarrow \infty}\left|E\left(r_{m}\right)\right| \geq \frac{2(q+1)}{\mu} \sin ^{-1}\left\{\frac{|\sin \pi \mu|}{(q+1)(1-\delta(0))}\right\},
$$

and the proof of $(11)$ is complete when $q$ is odd.

If $q$ is an even integer, we have to proceed slightly differently: Since $[(q+1) / 2]=q / 2$, the first sum in $(2.5)$ becomes

$$
\begin{aligned}
\mid \csc \pi & \mu \mid \sum_{j=0}^{q / 2}\left(\sin \mu \alpha_{2 j+1}-\sin \mu \alpha_{2 j}\right) \\
= & |\csc \pi \mu|\left\{\sin \mu \alpha_{1}+\sum_{j=1}^{q / 2} 2 \cos \frac{1}{2} \mu\left(\alpha_{2 j+1}+\alpha_{2 j}\right) \sin \frac{1}{2} \mu\left(\alpha_{2 j+1}-\alpha_{2 j}\right)\right\} \\
& \leq|\csc \pi \mu|\left\{\sin \mu \alpha_{1}+2 \sum_{j=1}^{q / 2} \sin \frac{1}{2} \mu\left(\alpha_{2 j+1}-\alpha_{2 j}\right)\right\},
\end{aligned}
$$

where the inequality is justified as in the case of odd $q$. We now let $x_{0}=\mu \alpha_{1}$, $x_{j}=\frac{1}{2} \mu\left(\alpha_{2 j+1}-\alpha_{2 j}\right)$ for $j=1, \ldots, q / 2$ and $x_{-j}=x_{j}$. This gives us $q+1$ numbers in $[0, \pi]$ to which we may apply the concavity of $\sin x$. We thus obtain

$$
\sin \left\{(q+1)^{-1}\left(x_{0}+\sum_{j=1}^{q / 2}\left(x_{j}+x_{-j}\right)\right)\right\} \geq(q+1)^{-1}\left\{\sin x_{0}+2 \sum_{j=1}^{q / 2} \sin x_{j}\right\} .
$$

It follows that

$$
\begin{aligned}
& \sin \mu \alpha_{1}+2 \sum_{j=1}^{q / 2} \sin \frac{1}{2} \mu\left(\alpha_{2 j+1}-\alpha_{2 j}\right) \\
& \quad \leq(q+1) \sin \left\{\frac{\mu \alpha_{1}+\sum_{j=1}^{q / 2} \mu\left(\alpha_{2 j+1}-\alpha_{2 j}\right)}{q+1}\right\} \\
& =(q+1) \sin \left\{\frac{\mu}{q+1}\left|C\left(r_{m}\right)\right|\right\} .
\end{aligned}
$$

Now (2.10) and (2.11) lead us back to (2.8) and (2.9). Hence (11) is true when $q$ is odd, and the proof of Theorem 1 is complete except for the statement about the sharpness of the inequalities.

\section{Proof of Theorem 2}

Assume that the lower order $\mu$ of $g$ satisfies

$$
q<\mu \leq q+\frac{1}{2}
$$


and that for infinitely many values of $m, \pi \in E\left(r_{m}\right)$. By taking a subsequence of $r_{m}$ which we also denote by $r_{m}$, we may assume that $\pi \in E\left(r_{m}\right)$ for all $m$. This means that we have

$$
\alpha_{q+1}\left(r_{m}\right)=\pi \text { for all } m \text {. }
$$

Now suppose that $q$ is even. Since $\alpha_{0}=0$, we may write

$$
\begin{aligned}
& |\csc \pi \mu| \sum_{j=0}^{[(q+1) / 2]}\left(\sin \mu \alpha_{2 j+1}-\sin \mu \alpha_{2 j}\right) \\
& \leq|\csc \pi \mu|\left\{2 \sum_{j=1}^{q / 2} \sin \frac{1}{2} \mu\left(\alpha_{2 j}-\alpha_{2 j-1}\right)+\sin \pi \mu\right\} \\
& \leq 1+2|\csc \pi \mu|(q / 2) \sin \left\{\frac{2}{q} \sum_{j=1}^{q / 2} \frac{1}{2} \mu\left(\alpha_{2 j}-\alpha_{2 j-1}\right)\right\} \\
& \quad=1+q|\csc \pi \mu| \sin \left\{\frac{\mu}{q} \sum_{j=1}^{q / 2}\left(\alpha_{2 j}-\alpha_{2 j-1}\right)\right\} .
\end{aligned}
$$

Recalling (18) and (3.2) we have, with $r=r_{m}$,

$$
\sum_{j=1}^{q / 2}\left(\alpha_{2 j}-\alpha_{2 j-1}\right)=\alpha_{q+1}-|C(r)|=\pi-|C(r)| .
$$

Now (3.3) and (3.4) used in (20) give

$$
1 \leq \sigma(1+o(1)) \cdot\left(1+q|\csc \pi \mu| \sin \left\{\frac{\pi \mu}{q}-\frac{\mu}{q}\left|C\left(r_{m}\right)\right|\right\}\right)+o(1)
$$

as $m \rightarrow \infty$.

Since $0 \leq y \leq \sin x$ for some $x$ in $[0, \pi]$ implies $\sin ^{-1} y \leq \pi-x$, we conclude from (3.5), with $x=\frac{\mu}{q}\left(\pi-\left|C\left(r_{m}\right)\right|\right)$, that

$$
\sin ^{-1}\left\{\frac{|\sin \pi \mu|}{q}\left(\frac{1+o(1)}{\sigma(1+o(1))}-1\right)\right\} \leq \pi-\left(\pi-\left|C\left(r_{m}\right)\right|\right) \frac{\mu}{q}
$$

and this readily yields (13) when $q$ is even.

If $q$ is odd, then (17) and (3.2) imply that

$$
\sum_{j=1}^{(q+1) / 2}\left(\alpha_{2 j}-\alpha_{2 j-1}\right)=\pi-\sum_{j=0}^{(q-1) / 2}\left(\alpha_{2 j+1}-\alpha_{2 j}\right) .
$$


Since $\alpha_{0}=\alpha_{q+2}=0$, the sum appearing in (20) may be written

$$
\begin{aligned}
\mid \csc & \pi \mu \mid \sum_{j=0}^{[(q+1) / 2]}\left(\sin \mu \alpha_{2 j+1}-\sin \mu \alpha_{2 j}\right) \\
& =|\csc \pi \mu|\left\{\sin \mu \alpha_{1}+\sum_{j=1}^{(q-1) / 2}\left(\sin \mu \alpha_{2 j+1}-\sin \mu \alpha_{2 j}\right)+|\sin \pi \mu|\right\} \\
& \leq|\csc \pi \mu|\left\{\sin \mu \alpha_{1}+2 \sum_{j=1}^{(q-1) / 2} \sin \frac{1}{2} \mu\left(\alpha_{2 j+1}-\alpha_{2 j}\right)+|\sin \pi \mu|\right\} \\
& \leq|\csc \pi \mu|\left\{q \sin \left\{\frac{\mu \alpha_{1}+2 \sum_{j=1}^{(q-1) / 2} \frac{1}{2} \mu\left(\alpha_{2 j+1}-\alpha_{2 j}\right)}{q}\right\}+|\sin \pi \mu|\right\} \\
& =|\csc \pi \mu|\left\{q \sin \left\{\frac{\mu}{q}\left(\pi-\left|C\left(r_{m}\right)\right|\right)\right\}+|\sin \pi \mu|\right\} \\
& =1+q|\csc \pi \mu| \sin \left\{\frac{\pi \mu}{q}-\frac{\mu}{q}\left|C\left(r_{m}\right)\right|\right\} .
\end{aligned}
$$

Thus the inequality (3.5) also holds true in the case of odd $q$, and hence (13) follows in this case also. Note that if $q=1$, the sum in the second line in (3.7) is empty.

\section{THE BEST POSSIBLE CHARACTER OF THEOREMS 1 AND 2}

Let $g$ be a Weierstrass canonical product of genus $q \geq 1$ and order $\mu$, $q<\mu<q+1$, having only real negative zeros and satisfying

$$
N(r)=N(r, 1 / g) \sim r^{\mu} L(r) \quad \text { as } r \rightarrow \infty,
$$

where $L$ is a slowly varying function. It is known [1] that

$$
\lim _{r \rightarrow \infty} \frac{1}{2 \pi} \int_{-\pi}^{\pi}\left|\frac{\log \left|g\left(r e^{i \theta}\right)\right|}{N(r)}-\psi_{\mu}(\theta)\right|^{p} d \theta=0 \quad(1 \leq p<\infty) .
$$

It follows from (4.2) that

$$
\lim _{r \rightarrow \infty} \frac{1}{2 \pi} \int_{-\pi}^{\pi}\left|\frac{\log ^{+}\left|g\left(r e^{i \theta}\right)\right|}{N(r)}-\psi_{\mu}^{+}(\theta)\right| d \theta=0,
$$

and also that

$$
\lim _{r \rightarrow \infty}\left|\int_{E(r)}\left(\frac{\log \left|g\left(r e^{i \theta}\right)\right|}{N(r)}-\psi_{\mu}(\theta)\right) d \theta\right|=0
$$

where $E(r)$ is any measurable subset of $[-\pi, \pi]$. 
Now (4.3) implies that $\lim _{r \rightarrow \infty} T(r, g) / N(r)=\frac{1}{\pi} \int_{0}^{\pi} \psi_{\mu}^{+}(\theta) d \theta$. If we take $E(r)$ to be the set defined in (4), then (4.4) implies

$$
\begin{aligned}
\lim _{r \rightarrow \infty} \phi(E(r)) & =\lim _{r \rightarrow \infty} \frac{1}{\pi} \int_{E(r)} \frac{\log \left|g\left(r e^{i \theta}\right)\right|}{N(r)} d \theta \\
& =\lim _{r \rightarrow \infty} \frac{2 T(r, g)}{N(r)}=\frac{2}{1-\delta(0)},
\end{aligned}
$$

and this shows that equality can hold in (10).

From (4.5) and (4.1), it follows that $T(r, g)$ is regularly varying and so every sequence is a sequence of Polya peaks of order $\mu$ of $T(r, g)$. Thus, by the remark after the proof of (2.4), we conclude that for such functions $g$,

$$
\alpha_{j}(r) \rightarrow \frac{(2 j-1)}{2 \mu} \pi \quad \text { as } r \rightarrow \infty
$$

for $j=1, \ldots,(q+1)$ if $q+\frac{1}{2} \leq \mu<q+1$; and for $j=1, \ldots, q$ and $\alpha_{q+1}(r) \rightarrow \pi$ if $q<\mu \leq q+\frac{1}{2}$. It follows that for such functions $g$, the measure of the set $E(r)$ defined in (4) satisfies

$$
|E(r)| \rightarrow\left|E_{\mu}\right|
$$

where $E_{\mu}=\left\{\theta \in[-\pi, \pi]: \psi_{\mu}(\theta) \geq 0\right\}$.

We can also compute $\delta(0)$ :

$$
\frac{1}{1-\overline{\delta(0)}}=\frac{1}{\pi} \int_{0}^{\pi} \psi_{\mu}^{+}(\theta) d \theta= \begin{cases}\frac{q+1}{|\sin \pi \mu|} & \text { if } q+\frac{1}{2} \leq \mu<q+1 \\ \frac{q+|\sin \pi \mu|}{|\sin \pi \mu|} & \text { if } q<\mu \leq q+\frac{1}{2} .\end{cases}
$$

It follows that

$$
\left|E_{\mu}\right|=\frac{(q+1) \pi}{\mu}=\frac{2(q+1)}{\mu} \sin ^{-1}\left\{\frac{|\sin \pi \mu|}{(q+1)(1-\delta(0))}\right\} \quad \text { if } q+\frac{1}{2}<\mu<q+1 \text {. }
$$

And if $q<\mu<q+\frac{1}{2}$, then

$$
\begin{aligned}
\left|E_{\mu}\right| & =\frac{(q-1) \pi}{\mu}+2\left(\pi-\frac{(2 q-1) \pi}{2 \mu}\right) \\
& =2 \pi\left(1-\frac{q}{\mu}\right)+\frac{(q-1) \pi}{\mu}+\frac{\pi}{\mu} \\
& =2 \pi\left(1-\frac{q}{\mu}\right)+\frac{q}{2 \mu} \sin ^{-1}\left(\frac{|\sin \pi \mu| \delta(0)}{q(1-\delta(0))}\right) .
\end{aligned}
$$

Now (4.7) and (4.8) imply that equality can hold in (13), which must therefore be a best possible inequality.

\section{REFERENCES}

1. F. F. Abi-Khuzam, An abelian theorem for a class of subharmonic functions, Proc. Amer. Math. Soc. 67 (1977), 253-259.

2. 82 (1981), 71-75. 
3. A. Baernstein II, Proof of Edrei's spread conjecture, Proc. London Math. Soc. 26 (1973), 418-434.

4. A. Edrei, The deficiencies of meromorphic functions of finite lower order, Duke Math. J. 31 (1964), 1-22.

5. S. Hellerstein and J. Williamson, Entire functions with negative zeros and a problem of $R$. Nevanlinna, J. Analyse Math. 22 (1969), 233-267.

Department of Mathematical Sciences, King Fahd University of Petroleum and Minerals, Dhahran 31261, Saudi Arabia

Current address: Department of Mathematics, American University of Beirut, Beirut, Lebanon 